\title{
Sodium and potassium intake and risk of cardiovascular events and all-cause mortality: the Rotterdam Study
}

\author{
Johanna M. Geleijnse · Jacqueline C. M. Witteman - Theo Stijnen • \\ Margot W. Kloos · Albert Hofman · Diederick E. Grobbee
}

Received: 2 August 2006/ Accepted: 12 September 2007/Published online: 28 September 2007

(C) Springer Science+Business Media B.V. 2007

\begin{abstract}
Background Dietary electrolytes influence blood pressure, but their effect on clinical outcomes remains to be established. We examined sodium and potassium intake in relation to cardiovascular disease (CVD) and mortality in an unselected older population. Methods A case-cohort analysis was performed in the Rotterdam Study among subjects aged 55 years and over, who were followed for 5 years. Baseline urinary samples were analyzed for sodium and potassium in 795 subjects who died, 206 with an incident myocardial infarction and 181 subjects with an incident stroke, and in 1,448 randomly selected subjects. For potassium, dietary data were additionally obtained by food-frequency questionnaire for $78 \%$ of the cohort. Results There was no consistent association of urinary sodium, potassium, or sodium/potassium ratio with CVD and all-cause mortality over the range of intakes observed in this population. Dietary potassium estimated by food frequency questionnaire, however, was associated with a lower risk of all-cause mortality in subjects initially free of $\mathrm{CVD}$ and hypertension $(\mathrm{RR}=0.71$ per standard deviation increase; 95\% confidence interval: 0.51-1.00). We observed a significant positive association between
\end{abstract}

J. M. Geleijnse ( $\square)$

Division of Human Nutrition, Wageningen University, P.O. Box 8129, 6700 EV Wageningen, The Netherlands e-mail: marianne.geleijnse@wur.nl

J. C. M. Witteman · T. Stijnen · M. W. Kloos · A. Hofman Department of Epidemiology \& Biostatistics, Erasmus MC Rotterdam, Rotterdam, The Netherlands

D. E. Grobbee

Julius Center for Health Sciences and Primary Care,

UMC Utrecht, Utrecht, The Netherlands urinary sodium/potassium ratio and all-cause mortality, but only in overweight subjects who were initially free of CVD and hypertension ( $\mathrm{RR}=1.19(1.02-1.39)$ per unit). Conclusion The effect of sodium and potassium intake on CVD morbidity and mortality in Western societies remains to be established.

Keywords Salt · Sodium · Potassium - Mortality · Cardiovascular disease - Myocardial infarction .

Stroke $\cdot$ Population-based

\section{Introduction}

Observational and experimental data support an independent, positive relationship between sodium intake and blood pressure, most clearly in hypertensive populations [1-3]. Potassium intake, on the other hand, has been inversely related to blood pressure [3, 4]. Since hypertension is a strong predictor of cardiovascular disease (CVD), especially stroke, inadequate intake of sodium and potassium is likely to be associated with increased cardiovascular morbidity and mortality [1]. Only recently, population-based studies on dietary salt intake in relation to CVD and non-cardiovascular events have received priority [5]. Alderman et al. were among the first to report an increased risk of myocardial infarction with low urinary sodium in treated hypertensive men [6]. In a subsequent analysis of NHANES I data, an inverse association of sodium intake with all-cause and cardiovascular mortality was found [7]. Estimation of salt intake by 24-h dietary recall and other methodological aspects of this analysis, however, have been criticized [8-10]. Salt intake was not significantly related to coronary or all-cause mortality in the large cohorts of the Scottish Heart Health Study [11] 
and the MRFIT trial [12]. A recent systematic review of 11 randomized trials showed no effect of long-term sodium reduction on overall mortality, but this meta-analysis included only 17 fatal events and should be interpreted with caution [13]. He et al. showed that high sodium intake was a strong risk factor for congestive heart failure in overweight participants of the NHANES I follow-up study [14], and also predictive for CVD and all-cause mortality in this group [15]. Similarly, in a Finnish cohort, 24-h urinary sodium excretion predicted mortality and risk of coronary heart disease only in the presence of overweight [16]. With regard to incidence of stroke, the Finnish study showed no association with urinary sodium [16]. Stroke mortality was neither predicted by dietary sodium intake in MRFIT [12]. In the WHO Cardiovascular Diseases and Alimentary Comparison (CARDIAC) Study in 24 countries, however, sodium intake appeared to be a risk factor for stroke in men [17]. As a consequence of these inconsistent findings, there is currently no consensus as to the cardiovascular risks of salt intake.

Tobian et al. demonstrated a lower risk of hemorrhagic stroke and mortality in hypertensive rats that had been given potassium supplements, an effect that was not mediated by blood pressure reduction [18]. Khaw and Barrett-Connor confirmed this independent protective effect of dietary potassium against stroke in humans [19]. Also in the CARDIAC study [17], the Cardiovascular Health Study [20] and the Nurses Health Study [21] the intake of potassium was inversely related to risk of stroke. Data on dietary potassium in relation to coronary and all-cause mortality in humans are scanty. We examined the relationship of sodium and potassium intake with cardiovascular events and allcause mortality in the older cohort of the populationbased Rotterdam Study.

\section{Methods}

\section{The Rotterdam Study}

This case-cohort analysis formed part of the Rotterdam Study, a population-based prospective study among 7,983 men and women aged 55 years and older in the Netherlands [22]. The Medical Ethics Committee of the Erasmus Medical Centre Rotterdam approved the study, and written informed consent was obtained from all participants. From August 1990 until June 1993, a trained research assistant collected data on health, medication use, lifestyle, and risk indicators for chronic diseases during a home interview. Subjects were subsequently invited at the study centre for clinical examination and assessment of diet.

\section{Assessment of diet}

Subjects were interviewed at the study centre by a trained dietician, who used a validated, semi-quantitative food frequency questionnaire [23]. The intake of total energy, alcohol, macronutrients, and a large number of micronutrients was computed using Dutch food composition tables [24]. No information on salt use was obtained and therefore data on dietary sodium were considered unreliable for this analysis.

\section{Clinical examination}

Height and body weight were measured with the subject wearing indoor clothing without shoes. The body mass index was computed as weight divided by height squared. A trained research assistant measured sitting systolic and diastolic blood pressure twice with a random-zero sphygmomanometer after a 5-min rest, and values were averaged. Hypertension was defined as a systolic blood pressure $\geq 160 \mathrm{mmHg}$ or diastolic blood pressure $\geq 95 \mathrm{mmHg}$ or use of antihypertensive medication. Diabetes mellitus was considered present when the subject reported antidiabetic treatment, or when random or postload plasma glucose levels were $11.1 \mathrm{mmol} / \mathrm{l}$ or higher. CVD was considered present in case of a verified history of myocardial infarction, stroke, coronary bypass grafting, or percutaneous transluminal coronary angioplasty. Serum total and HDL cholesterol level ( $\mathrm{mmol} / \mathrm{l})$ were determined by standard laboratory methods [25].

\section{Assessment of sodium and potassium excretion}

Participants collected an overnight urine sample before visiting the research centre and recorded collection times on the jar. They were not aware that samples would be used for estimation of electrolyte intake. At the research centre, volumes were recorded, urines were swirled and $100 \mathrm{ml}$ samples were taken. Samples were stored in plastic tubes at $-20^{\circ} \mathrm{C}$ for future laboratory determinations. Urinary sodium, potassium and creatinine determinations were performed by $\operatorname{Vitros}^{\circledR} 250$ (formerly Ektachem 250) Chemistry System (Johnson \& Johnson, Ortho-Clinical Diagnostics Inc., Rochester, New York). Determination of electrolytes and creatinine were based on potentiometry and enzymatic conversion, respectively. Urinary sodium and potassium concentrations $(\mathrm{mmol} / \mathrm{l})$ were standardized to 24-h values using recorded collection times and urinary volumes $(\mathrm{ml})$. In addition, urinary sodium/potassium ratio was computed. 
Follow-up procedures

The present analysis is based on follow-up data collected from baseline (1990-1993) until 1 January 1998. Informed consent for collection of follow-up data was obtained from 7,802 participants (98\%). Information on vital status was obtained at regular intervals from municipal population registries. General practitioners (GPs) used a computerized information system to record fatal and non-fatal events in the research area (covering $85 \%$ of the cohort). In the Netherlands, the GP forms the link to all specialized medical care and clinical events are unlikely to be missed by this follow-up procedure. Research physicians verified all information on incident events using GP records and hospital discharge letters. Events were coded independently by two physicians according to the International Classification of Diseases, 10th revision (ICD-10) [26]. Coded events were reviewed by a medical expert in the field, whose judgment was considered definite in case of discrepancies.

Myocardial infarction comprised ICD-10 code I21 and stroke comprised ICD-10 codes I60-I67. Both fatal and nonfatal incident events were recorded. For the present study, only first events were considered. Events followed by death within 28 days were classified as fatal. CVD mortality comprised fatal myocardial infarction, fatal stroke, sudden cardiac death and other forms of fatal CVD (ICD-10 codes I20-I25, I46, I49, I50, I60-I67, I70-I74, and R96).

Table 1 Baseline characteristics of the study population

\begin{tabular}{|c|c|c|c|c|c|}
\hline & \multirow[t]{2}{*}{ Random sample } & \multicolumn{4}{|l|}{ Cases } \\
\hline & & Incident MI & Incident stroke & CVD mortality & All-cause mortality \\
\hline No. of subjects & 1,448 & 206 & 181 & 217 & 795 \\
\hline In random sample (\%) & & 31 & 31 & 28 & 29 \\
\hline Age (year) & $69.2(8.7)$ & $71.0(8.0)$ & $74.0(8.5)$ & $76.8(8.4)$ & $76.9(8.9)$ \\
\hline Men $(\%)$ & 41 & 62 & 45 & 51 & 49 \\
\hline Body mass index $\left(\mathrm{kg} / \mathrm{m}^{2}\right)$ & $26.4(3.8)$ & $26.3(3.4)$ & $26.0(3.3)$ & $26.2(3.8)$ & $25.7(3.8)$ \\
\hline \multicolumn{6}{|l|}{ Smoking status $(\%)^{\mathrm{a}}$} \\
\hline Current & 23 & 29 & 28 & 23 & 26 \\
\hline Former & 41 & 48 & 42 & 47 & 40 \\
\hline Never & 36 & 23 & 29 & 29 & 35 \\
\hline Alcohol use (\%) & 81 & 74 & 80 & 71 & 73 \\
\hline \multicolumn{6}{|l|}{ Educational level $(\%)^{\mathrm{a}, \mathrm{b}}$} \\
\hline Low & 58 & 61 & 60 & 65 & 66 \\
\hline Intermediate & 32 & 31 & 34 & 30 & 28 \\
\hline High & 10 & 8 & 6 & 5 & 6 \\
\hline \multicolumn{6}{|l|}{ Serum cholesterol $(\mathrm{mmol} / \mathrm{l})$} \\
\hline Total & $6.6(1.2)$ & $6.3(1.3)$ & $6.5(1.2)$ & $6.6(1.4)$ & $6.3(1.3)$ \\
\hline HDL & $1.4(0.4)$ & $1.3(0.4)$ & $1.3(0.4)$ & $1.2(0.4)$ & $1.3(0.4)$ \\
\hline \multicolumn{6}{|l|}{ Blood pressure (mmHg) } \\
\hline Systolic & $140(22)$ & $145(23)$ & $149(24)$ & $146(25)$ & $145(25)$ \\
\hline Diastolic & $74(11)$ & $74(12)$ & $75(13)$ & $73(13)$ & $73(14)$ \\
\hline Hypertension $(\%)^{\mathrm{c}}$ & 37 & 44 & 53 & 55 & 47 \\
\hline Diabetes mellitus $(\%)^{\mathrm{d}}$ & 10 & 21 & 22 & 26 & 21 \\
\hline History of CVD (\%) & 17 & 35 & 17 & 39 & 28 \\
\hline
\end{tabular}

Values are means with standard deviations, or percentages; CVD, cardiovascular disease; MI, myocardial infarction

${ }^{\text {a }}$ Values not always add up to $100 \%$ due to rounding

${ }^{\mathrm{b}}$ Highest achieved level of education; low, primary education, or less; intermediate, secondary general or vocational education; high, higher vocational education, university

c Systolic blood pressure $\geq 160 \mathrm{mmHg}$ or diastolic blood pressure $\geq 95 \mathrm{mmHg}$ or use of antihypertensive medication

${ }^{\mathrm{d}}$ Plasma glucose $\geq 11.1 \mathrm{mmol} / \mathrm{l}$ or treated with oral antidiabetes medication or insulin

e Verified history of cardiovascular disease, i.e. myocardial infarction, stroke, coronary bypass-grafting, or percutaneous transluminal coronary angioplasty 
Table 2 Baseline urinary excretions and dietary intakes of Dutch men and women aged 55 years and over: The Rotterdam Study

\begin{tabular}{|c|c|c|c|c|c|}
\hline & \multirow[t]{2}{*}{ Random subcohort } & \multicolumn{4}{|l|}{ Cases } \\
\hline & & Incident MI & Incident stroke & CVD mortality & All-cause mortality \\
\hline \multicolumn{6}{|l|}{ Urinary excretion $^{\mathrm{a}}$} \\
\hline Volume (1/24 h) & $1.4(0.6)$ & $1.4(0.6)$ & $1.4(0.6)$ & $1.3(0.6)$ & $1.3(0.6)$ \\
\hline Sodium (mmol/24 h) & $117(69)$ & $124(68)$ & $115(72)$ & $99(61)$ & 107 (66) \\
\hline Potassium (mmol/24 h) & $45(22)$ & $47(22)$ & $45(23)$ & $44(24)$ & $44(22)$ \\
\hline Sodium/potassium & $2.8(1.5)$ & $2.7(1.3)$ & $2.7(1.3)$ & $2.5(1.4)$ & $2.6(1.6)$ \\
\hline Creatinine $(\mathrm{mmol} / 24 \mathrm{~h})$ & $9.2(4.9)$ & $9.8(4.7)$ & $8.4(4.4)$ & $8.1(4.7)$ & $8.1(4.4)$ \\
\hline Sodium/creatinine & $13.8(6.6)$ & $13.6(6.1)$ & $14.6(7.1)$ & $14.0(8.0)$ & $14.8(7.9)$ \\
\hline Potassium/creatinine & $5.4(2.2)$ & $5.3(2.1)$ & $5.8(2.1)$ & $6.1(2.6)$ & $6.1(2.5)$ \\
\hline \multicolumn{6}{|l|}{ Dietary intake $e^{\mathrm{b}}$} \\
\hline Total energy (mJ/day) & $8.3(2.1)$ & $8.6(2.2)$ & $8.4(2.2)$ & $8.3(2.0)$ & $8.5(2.2)$ \\
\hline Saturated fat (g/day) & $32(12)$ & $34(13)$ & $34(13)$ & $33(13)$ & $34(12)$ \\
\hline Calcium (g/day) & $1.1(0.4)$ & $1.1(0.4)$ & $1.1(0.4)$ & $1.1(0.5)$ & $1.1(0.4)$ \\
\hline Sodium $(\mathrm{g} / \mathrm{day})^{\mathrm{c}}$ & $2.2(0.7)$ & $2.3(0.6)$ & $2.2(0.6)$ & $2.2(0.7)$ & $2.2(0.7)$ \\
\hline Potassium (g/day) & $3.6(0.8)$ & $3.7(0.8)$ & $3.6(0.8)$ & $3.6(0.9)$ & $3.6(0.9)$ \\
\hline
\end{tabular}

Values are means with standard deviations; CVD, cardiovascular disease; MI, myocardial infarction

a Based on one timed overnight urine sample

b Dietary data were available for 1,205 subjects of the random sample (83\%), 170 MI cases (83\%), 147 stroke cases (81\%), 157 CVD deaths $(72 \%)$, and 518 deaths from any cause $(65 \%)$

c Only from foods, discretionary sources not included

Study population

Of 7,129 subjects who visited the research centre, 6,605 adequately performed a timed overnight urine collection for which collection times were recorded and volumes exceeded $150 \mathrm{ml}$. Of those, 5,531 had blood pressure readings and these subjects were eligible for the present analysis. We followed a case-cohort approach for efficiency reasons. Assessment of urinary sodium, potassium and creatinine excretion was performed in all subjects who died ( $n=795$, including 217 cardiovascular deaths), and in those who experienced a myocardial infarction $(n=206)$ or stroke $(n=181)$ during follow-up. A random sample of 1,500 control subjects was taken from the eligible cohort for assessment of electrolyte excretions. Urine samples could not be retrieved for 52 of these subjects, and data on urinary sodium, potassium and creatinine were thus obtained in 1,448 subjects. Dietary data were available for 1,205 subjects $(83 \%)$ of the random sample, 518 subjects $(65 \%)$ who died during follow-up, 157 subjects $(72 \%)$ who died from CVD, 170 subjects $(83 \%)$ with an incident myocardial infarction and 147 subjects $(81 \%)$ with an incident stroke. Reasons for missing dietary data were participation in the pilot phase of the Rotterdam Study, low cognitive function, and logistic reasons, as described in more detail elsewhere [23]. Of the random sub-cohort $(n=1,448), 783$ subjects $(54 \%)$ were free of CVD and hypertension at baseline.
Data analysis

Pearson correlations were computed to examine interrelationships between urinary and dietary measures of electrolyte intake and associations with total energy intake.

The association of urinary and dietary electrolytes with incident myocardial infarction, incident stroke, cardiovascular mortality and all-cause mortality was evaluated in a case-cohort design with standard Cox proportional-hazards models with modification of the standard errors based on robust variance estimates [27, 28]. We used the method according to Barlow in which the random cohort is weighted by the inverse of the sampling fraction from the source population. Members of the random cohort are included from baseline until failure or censoring, whereas cases outside the cohort are included at the time of their event. For the Cox models we used Proc MI and Proc MIanalyze, in conjunction with Proc Phreg (SAS 8.2).

Relative risks (RR) with $95 \%$ confidence intervals (95\%-CI) were computed per 1 standard deviation increase in urinary sodium $(\mathrm{mmol} / 24 \mathrm{~h})$, urinary potassium $(\mathrm{mmol} /$ $24 \mathrm{~h}$ ) and dietary potassium intake (mg/day), and per 1 unit increase in urinary sodium/potassium ratio. Two-sided $P$ values below 0.05 were considered statistically significant. Adjustment was made for age, sex and, in urinary analyses, for 24-h urinary creatinine excretion (model 1). In a second analysis (model 2), additional adjustment was made for body mass index $\left(\mathrm{kg} / \mathrm{m}^{2}\right)$, smoking status (current, past, or 
never), diabetes mellitus (yes/no), use of diuretics (yes/no), and highest completed education (three categories). In a third analysis (model 3), dietary confounders were additionally adjusted for, i.e. daily intake of total energy $(\mathrm{kJ})$, alcohol $(\mathrm{g})$, calcium $(\mathrm{g})$, and saturated fat $(\mathrm{g})$. In the analysis for urinary sodium we additionally included urinary potassium in this model, and vice versa.

Analyses were repeated after exclusion of subjects with a history of CVD or hypertension to avoid biased risk estimates due to intentional dietary changes. Within this sub-cohort, a predefined stratified analysis of urinary sodium and urinary sodium/potassium ratio with cardiovascular and all-cause mortality was performed in subjects with a high body mass index (i.e., $\geq 25 \mathrm{~kg} / \mathrm{m}^{2}$ ), using model 3.

Also in the sub-cohort free of CVD and hypertension, the distribution of 24-h urinary sodium excretion was

Table 3 Relative risk of urinary sodium with cardiovascular events and all-cause mortality in Dutch men and women aged 55 years and over

\begin{tabular}{|c|c|c|}
\hline & All subjects ${ }^{a}$ & $\begin{array}{l}\text { Subjects initially free } \\
\text { of CVD and hypertension }\end{array}$ \\
\hline \multicolumn{3}{|l|}{ Incident $M I$} \\
\hline $\mathrm{RR}$, model $1^{\mathrm{b}}$ & $1.13(0.95-1.34)$ & $1.04(0.75-1.43)$ \\
\hline $\mathrm{RR}$, model $2^{\mathrm{c}}$ & $1.16(0.98-1.39)$ & $1.07(0.77-1.50)$ \\
\hline $\mathrm{RR}$, model $3^{\mathrm{d}}$ & $1.19(0.97-1.46)$ & $1.14(0.77-1.69)$ \\
\hline \multicolumn{3}{|l|}{ Incident stroke } \\
\hline RR, model 1 & $1.09(0.89-1.33)$ & $1.16(0.84-1.61)$ \\
\hline RR, model 2 & $1.09(0.87-1.35)$ & $1.15(0.81-1.62)$ \\
\hline RR, model 3 & $1.08(0.80-1.46)$ & $1.02(0.66-1.58)$ \\
\hline \multicolumn{3}{|l|}{ CVD mortality ${ }^{\mathrm{e}}$} \\
\hline RR, model 1 & $0.74(0.60-0.91)$ & $0.84(0.59-1.22)$ \\
\hline RR, model 2 & $0.83(0.68-1.02)$ & $0.95(0.66-1.39)$ \\
\hline RR, model 3 & $0.77(0.60-1.01)$ & $0.83(0.47-1.44)$ \\
\hline \multicolumn{3}{|c|}{ All-cause mortality } \\
\hline RR, model 1 & $0.90(0.81-1.02)$ & $1.00(0.83-1.20)$ \\
\hline RR, model 2 & $0.96(0.84-1.09)$ & $1.10(0.91-1.34)$ \\
\hline RR, model 3 & $0.95(0.81-1.12)$ & $1.12(0.86-1.46)$ \\
\hline
\end{tabular}

RR, Relative risk with $95 \%$ confidence interval per standard deviation increase in urinary sodium $(\mathrm{mmol} / 24 \mathrm{~h})$, obtained by Cox proportional hazard analysis

a Number of cases and subjects in random sample given in Table 1

b Ajusted for age, sex and (for urinary sodium) 24-h urinary creatinine excretion

c As model 1, with additional adjustment for body mass index, smoking status, diabetes, use of diuretics, highest completed education

d As model 2, with additional adjustment for daily intake of total energy, alcohol, calcium, saturated fat and 24-h urinary potassium excretion

e Cardiovascular mortality comprises fatal myocardial infarction, fatal stroke, sudden cardiac death and other forms of fatal CVD divided into quartiles to be able to examine the relationship with all-cause mortality at extreme intakes. Quartiles of urinary sodium (cut-off levels: 66, 105 and $151 \mathrm{mmol} /$ $24 \mathrm{~h}$ ) were entered categorically into the fully adjusted model (model 3), using the lower quartile as the reference.

\section{Results}

The study had a median follow-up of 5.5 years. Baseline characteristics of the study population are shown in Table 1 . Randomly selected controls $(n=1,448)$ were expectedly healthier at baseline than cases, as indicated by a lower prevalence of hypertension, diabetes, and CVD.

Baseline urinary excretions and dietary intakes are presented in Table 2. In the random sample, 24-h urinary sodium excretion estimated from overnight urine collection was $117 \mathrm{mmol}$ (i.e., $2.7 \mathrm{~g} / \mathrm{day}$, which corresponds to a $\mathrm{NaCl}$ intake of $6.8 \mathrm{~g} /$ day). Urinary potassium excretion was $45 \mathrm{mmol} / 24 \mathrm{~h}(1.8 \mathrm{~g} / \mathrm{day})$, which was half the amount estimated by food frequency questionnaire ( $3.6 \mathrm{~g} /$ day $)$. The correlation between urinary and dietary potassium was 0.21 $(P<0.001)$.

RR for cardiovascular events and all-cause mortality per 1 -SD increase in 24-h urinary sodium are presented in Table 3. Urinary sodium was not significantly associated with incident myocardial infarction, incident stroke, or overall mortality. For CVD mortality, however, a borderline significant inverse association was observed $(\mathrm{RR}=0.77$ (0.60-1.01) per 1-SD, model 3) but the relationship was attenuated after excluding subjects with a history of CVD or hypertension $(\mathrm{RR}=0.83(0.47-1.44)$ per 1-SD, model 3). In subjects initially free of CVD, the risk of all-cause mortality was also examined across quartiles of 24-h urinary sodium (median values: 45, 87, 125 and $190 \mathrm{mmol}$, respectively). RR in consecutive quartiles, using the lower quartile as the reference, were 0.80 (0.43-1.49), 0.66 (0.34-1.27) and 0.98 (0.54-1.78), respectively (model 3 ). In a subgroup analysis of CVD free subjects with a body mass index $\geq 25 \mathrm{~kg} / \mathrm{m}^{2}$, the association of urinary sodium with CVD mortality or all-cause mortality was neither statistically significant $(\mathrm{RR}=0.91(0.44-$ 1.89) and $\mathrm{RR}=1.19(0.86-1.66)$ per $1-\mathrm{SD}$, respectively; model 3).

Findings for potassium are presented in Table 4. Urinary potassium tended to be positively associated with incident CVD events or mortality, especially in subjects who were initially free of CVD and hypertension. After full adjustment for confounders (model 3), however, none of these associations were statistically significant. Urinary potassium did neither predict all-cause mortality. For dietary potassium, similar results were obtained except for risk of all-cause mortality that was significantly reduced both in 
Table 4 Relationship of urinary and dietary potassium with cardiovascular events and all-cause mortality in Dutch men and women aged 55 years and over

\begin{tabular}{|c|c|c|c|c|}
\hline & \multicolumn{2}{|l|}{ All subjects ${ }^{\mathrm{a}}$} & \multicolumn{2}{|c|}{ Subjects initially free of CVD and hypertension ${ }^{\mathrm{a}}$} \\
\hline & $\begin{array}{l}\text { Urinary excretion } \\
(\mathrm{mmol} / 24 \mathrm{~h})\end{array}$ & $\begin{array}{l}\text { Dietary intake } \\
(\mathrm{mg} / \text { day })\end{array}$ & $\begin{array}{l}\text { Urinary excretion } \\
(\mathrm{mmol} / 24 \mathrm{~h})\end{array}$ & $\begin{array}{l}\text { Dietary intake } \\
\text { (mg/day) }\end{array}$ \\
\hline \multicolumn{5}{|l|}{ Incident MI } \\
\hline $\mathrm{RR}$, model $1^{\mathrm{b}}$ & $1.10(0.89-1.35)$ & $0.98(0.85-1.13)$ & $1.15(0.84-1.59)$ & $1.14(0.85-1.54)$ \\
\hline $\mathrm{RR}$, model $2^{\mathrm{c}}$ & $1.16(0.94-1.43)$ & $0.94(0.81-1.09)$ & $1.25(0.94-1.74)$ & $1.07(0.78-1.46)$ \\
\hline RR, model $3^{\mathrm{d}}$ & $1.11(0.87-1.43)$ & $0.90(0.65-1.24)$ & $1.22(0.79-1.87)$ & $1.32(0.65-2.67)$ \\
\hline \multicolumn{5}{|l|}{ Incident stroke } \\
\hline RR, model 1 & $1.09(0.87-1.36)$ & $0.99(0.84-1.17)$ & $1.12(0.79-1.60)$ & $1.07(0.79-1.43)$ \\
\hline RR, model 2 & $1.12(0.89-1.42)$ & $0.99(0.84-1.16)$ & $1.15(0.77-1.71)$ & $1.20(0.86-1.68)$ \\
\hline RR, model 3 & $1.17(0.86-1.58)$ & $1.02(0.71-1.46)$ & $1.11(0.61-2.04)$ & $1.06(0.50-2.29)$ \\
\hline \multicolumn{5}{|c|}{ CVD mortality ${ }^{\mathrm{e}}$} \\
\hline RR, model 1 & $1.13(0.90-1.41)$ & $0.97(0.82-1.14)$ & $1.63(1.14-2.33)$ & $1.23(0.83-1.84)$ \\
\hline RR, model 2 & $1.14(0.92-1.42)$ & $0.95(0.81-1.12)$ & $1.66(1.08-2.56)$ & $1.19(0.78-1.83)$ \\
\hline RR, model 3 & $1.23(0.94-1.60)$ & $0.97(0.72-1.31)$ & $1.45(0.84-2.54)$ & $1.43(0.67-3.03)$ \\
\hline \multicolumn{5}{|c|}{ All-cause mortality } \\
\hline RR, model 1 & $1.04(0.91-1.18)$ & $0.91(0.82-1.01)$ & $1.06(0.88-1.28)$ & $0.95(0.78-1.17)$ \\
\hline RR, model 2 & $1.06(0.86-1.31)$ & $0.89(0.80-0.99)$ & $1.06(0.86-1.31)$ & $0.90(0.73-1.12)$ \\
\hline RR, model 3 & $1.08(0.91-1.28)$ & $0.78(0.65-0.94)$ & $0.95(0.71-1.26)$ & $0.71(0.51-1.00)$ \\
\hline \multicolumn{5}{|c|}{$\begin{array}{l}\text { RR, Relative risk with } 95 \% \text { confidence interval per standard deviation increase in urinary or dietary potassium, obtained by Cox proportiona } \\
\text { hazard analysis }\end{array}$} \\
\hline \multicolumn{5}{|c|}{ a Number of cases and subjects in random sample given in Table 1} \\
\hline \multicolumn{5}{|c|}{ b Ajusted for age, sex and (for urinary potassium) 24-h urinary creatinine excretion } \\
\hline \multicolumn{5}{|c|}{ ' ' As model 1, with additional adjustment for body mass index, smoking status, diabetes, use of diuretics and highest completed education } \\
\hline \multicolumn{5}{|c|}{ d As model 2, with additional adjustment for daily intake of total energy, alcohol, calcium, saturated fat and 24-h urinary sodium excretion } \\
\hline
\end{tabular}

the entire cohort $(\mathrm{RR}=0.78(0.65-0.94$ per $1-\mathrm{SD})$ and in subjects initially free of $\mathrm{CVD}$ and hypertension $(\mathrm{RR}=0.71$ (0.51-1.00), model 3).

Data for urinary sodium/potassium ratio (Table 5) showed no relationship with CVD events and mortality. When restricting this analysis to CVD free subjects with a body mass index $\geq 25 \mathrm{~kg} / \mathrm{m}^{2}$, urinary sodium/potassium ratio was significantly associated with all-cause mortality $(\mathrm{RR}=1.19$ (1.02-1.39) per unit, model 3), but not with CVD mortality $(\mathrm{RR}=0.86(0.60-1.25))$.

\section{Discussion}

In an unselected population of older Dutch subjects we found no consistent association of urinary sodium and potassium with CVD events or mortality. Dietary potassium estimated by food frequency questionnaire, however, was associated with a lower risk of all-cause mortality. Urinary sodium/potassium ratio was positively associated with mortality risk, but only in overweight subjects without CVD and hypertension at baseline.
Electrolyte intake was assessed from one overnight urine collection, which provides a crude estimate of shortterm intake [29,30]. Luft et al. examined the utility of nocturnal sodium excretion under controlled intake conditions, in which daily sodium intake was randomly varied [31]. In that study, on a randomly selected day, both 24-h and nocturnal sodium excretion estimated the daily intake reasonably well. Nevertheless, it is likely that misclassification has attenuated the relationships with CVD events and mortality in our study. Incomplete urine collection was partly adjusted for by adding urinary creatinine excretion to the multivariate models. In addition, we examined the urinary sodium/potassium ratio, which is less influenced by incomplete urine collection. To exclude bias due to dietary changes, we repeated all analyses in a subgroup without CVD or hypertension at baseline.

Salt intake was not consistently related to CVD or mortality in our study. An explanation for the absence of a positive relationship, apart from regression dilution bias, may be the relatively narrow range of salt intake in the Netherlands and the lack of contrast in exposure within a single population. An increased risk of mortality was 
Table 5 Relationship of urinary sodium/potassium ratio with cardiovascular events and all-cause mortality in Dutch men and women aged 55 years and over

$\begin{array}{ll}\text { All subjects }^{\mathrm{a}} & \begin{array}{l}\text { Subjects initially free } \\ \text { of CVD and hypertension }\end{array}\end{array}$

\begin{tabular}{|c|c|c|}
\hline \multicolumn{3}{|l|}{ Incident MI } \\
\hline RR, model $1^{\mathrm{b}}$ & $1.03(0.93-1.14)$ & $0.92(0.76-1.13)$ \\
\hline $\mathrm{RR}$, model $2^{\mathrm{c}}$ & $1.02(0.92-1.13)$ & $0.90(0.73-1.10)$ \\
\hline RR, model $3^{\mathrm{d}}$ & $1.04(0.93-1.17)$ & $0.91(0.72-1.16)$ \\
\hline \multicolumn{3}{|l|}{ Incident stroke } \\
\hline RR, model 1 & $1.01(0.89-1.13)$ & $1.01(0.83-1.23)$ \\
\hline RR, model 2 & $0.99(0.86-1.13)$ & $0.99(0.77-1.20)$ \\
\hline RR, model 3 & $0.99(0.83-1.18)$ & $0.90(0.66-1.22)$ \\
\hline \multicolumn{3}{|l|}{ CVD mortality ${ }^{\mathrm{e}}$} \\
\hline RR, model 1 & $0.88(0.77-1.01)$ & $0.85(0.65-1.11)$ \\
\hline RR, model 2 & $0.93(0.81-1.06)$ & $0.86(0.66-1.13)$ \\
\hline RR, model 3 & $0.92(0.80-1.07)$ & $0.91(0.65-1.27)$ \\
\hline \multicolumn{3}{|c|}{ All-cause mortality } \\
\hline RR, model 1 & $0.99(0.91-1.06)$ & $1.04(0.91-1.18)$ \\
\hline RR, model 2 & $0.99(0.92-1.08)$ & $1.06(0.93-1.22)$ \\
\hline RR, model 3 & $1.01(0.91-1.12)$ & $1.13(0.93-1.36)$ \\
\hline
\end{tabular}

RR, Relative risk with $95 \%$ confidence interval per 1 unit increase in urinary sodium/potassium ratio, obtained by Cox proportional hazard analysis

a Number of cases and subjects in random sample given in Table 1

b Adjusted for age, sex and 24-h urinary creatinine excretion

c As model 1, with additional adjustment for body mass index, smoking status, diabetes, use of diuretics and highest completed education

d As model 2, with additional adjustment for daily intake of total energy, alcohol, calcium, and saturated fat

e Cardiovascular mortality comprises fatal myocardial infarction, fatal stroke, sudden cardiac death and other forms of fatal CVD

observed for high salt intake in overweight Finnish subjects with 24-h urinary excretions close to $200 \mathrm{mmol}(\mathrm{RR}=$ 1.56 per $100 \mathrm{mmol}$ ) [16]. However, this could not be confirmed in our analysis of quartiles of sodium intake in relation to overall mortality $(\mathrm{RR}=0.98$ in CVD free subjects with median sodium excretion of $190 \mathrm{mmol} / 24 \mathrm{~h}$ ). The absence of a relationship between salt intake and mortality in our study corroborates the findings from the large Scottish Heart Health Study among almost 12,000 middle-aged subjects with 24-h urine samples [11]. Follow-up data of the MRFIT trial neither showed a relationship between dietary sodium intake estimated by 24-h recall and cardiovascular events or mortality [12]. However, other prospective epidemiological studies do suggest that sodium intake is related to morbidity and mortality [6, 7, 15, 16], although this may be confined to specific subgroups with overweight, hypertension or high salt intake. In overweight subjects, we did find a positive relationship between urinary sodium/potassium ratio and overall mortality $(19 \%$ increase in risk per unit change in sodium/potassium ratio).

A protective effect of potassium intake against stroke, as previously reported [19-21], could not be confirmed by our data. We neither observed an association between potassium intake and coronary events. Long-term rather than short-term intake may be relevant and therefore we estimated habitual potassium intake during the preceding year by food frequency questionnaire. Mortality risk was reduced by $29 \%$ per 1 -SD increase in dietary potassium, although only in subjects initially free of CVD and hypertension. Except for misclassification, although less likely than for sodium, we have no explanation for the absent relationship between potassium intake and CVD events. In the Scottish Heart Health Study, 24-h urinary potassium excretion was inversely related to all-cause mortality and coronary events [11]. Data on potassium intake in relation to mortality, however, are sparse and more prospective population-based studies are needed to draw conclusions. Preferably, the effect of dietary potassium on CVD should be examined in a randomized trial.

Prolonged differences in blood pressure of $5 \mathrm{mmHg}$ may result in a one-third reduction in stroke and one-fifth reduction in coronary events [32]. Meta-analysis of randomized controlled trials showed that sodium reduction around $2 \mathrm{~g}$ per day could lower blood pressure by $2-$ $3 \mathrm{mmHg}$, with the effect being twice as large in hypertensives [33]. The World Health Organization recommends that people should consume less than $5 \mathrm{~g}$ of salt (i.e. $2 \mathrm{~g}$ of sodium) per day in order to prevent CVD [34]. From this and other epidemiological studies we conclude that effect of dietary salt on clinical cardiovascular endpoints and overall mortality within the range of intake commonly observed in Western countries has not yet been established. More research is needed to settle the discussion regarding this major public health issue.

Acknowledgment This work was funded by the Dutch Kidney Foundation (Grant\# C99-1847).

\section{References}

1. MacMahon S, Peto R, Cutler J, et al. Blood pressure, stroke, and coronary heart disease. Part 1, prolonged differences in blood pressure: prospective observational studies corrected for the regression dilution bias. Lancet 1990;335:765-74.

2. Graudal NA, Galloe AM, Garred P. Effects of sodium restriction on blood pressure, renin, aldosterone, catecholamines, cholesterols, and triglyceride: a meta-analysis. JAMA 1998;279:138391.

3. Grobbee DE. Electrolyte intake and human hypertension: sodium and potassium. In: Swales JD, editor. Textbook of hypertension. Oxford: Blackwell Scientific Publications; 1994. p. 539-51. 
4. Geleijnse JM, Kok FJ, Grobbee DE. Blood pressure response to changes in sodium and potassium intake: a metaregression analysis of randomised trials. J Hum Hypertens 2003;17:471-80.

5. Perry IJ. Dietary salt intake and cerebrovascular damage. Nutr Metab Cardiovasc Dis 2000;10:229-35.

6. Alderman MH, Madhavan S, Cohen $\mathrm{H}$, et al. Low urinary sodium is associated with greater risk of myocardial infarction among treated hypertensive men. Hypertension 1995;25:1144-52.

7. Alderman $\mathrm{MH}$, Cohen $\mathrm{H}$, Madhavan S. Dietary sodium intake and mortality: the National Health and Nutrition Examination Survey (NHANES I). Lancet 1998;351:781-5.

8. De Wardener H, MacGregor GA. Sodium intake and mortality. Lancet 1998;351:1508.

9. Engelman K. Sodium intake and mortality. Lancet 1998; 351:1508-9.

10. Karppanen H, Mervaala E. Sodium intake and mortality. Lancet 1998;351:1509.

11. Tunstall-Pedoe H, Woodward M, Tavendale R, et al. Comparison of the prediction by 27 different factors of coronary heart disease and death in men and women of the Scottish heart health study: cohort study. BMJ 1997;315:722-9.

12. Cohen JD, Grandits G, Cutler JA, et al. Dietary sodium intake and mortality: MRFIT follow-up study results [abstract]. Circulation 1999;100(Suppl I): I-524.

13. Hooper L, Bartlett C, Davey Smith G, et al. Systematic review of long term effects of advice to reduce dietary salt in adults. BMJ 2002;325:628-37.

14. He J, Ogden LG, Bazzano LA, et al. Dietary sodium intake and incidence of congestive heart failure in overweight US men and women: first National Health and Nutrition Examination Survey Epidemiologic Follow-up Study. Arch Intern Med 2002; 162:1619-24.

15. He J, Ogden LG, Vupputuri S, et al. Dietary sodium intake and subsequent risk of cardiovascular disease in overweight adults. JAMA 1999;282:2027-34.

16. Tuomilehto J, Jousilahti P, Rastenyte D, et al. Urinary sodium excretion and cardiovascular mortality in Finland: a prospective study. Lancet 2001;357:848-51.

17. Yamori Y, Nara Y, Mizushima S, et al. Nutritional factors for stroke and major cardiovascular diseases: international epidemiological comparison of dietary prevention. Health Rep 1994; 6:22-7.

18. Tobian L, Lange J, Ulm K, et al. Potassium reduces cerebral hemorrhage and death rate in hypertensive rats, even when blood pressure is not lowered. Hypertension 1985;7(Suppl I):I-110-I-114.

19. Khaw KT, Barrett-Connor E. Dietary potassium and strokeassociated mortality. N Engl J Med 1987;316:235-40.
20. Green DM, Ropper AH, Kronmal RA, et al. The Cardiovascular Health Study. Serum potassium level and dietary potassium intake as risk factors for stroke. Neurology 2002;59:314-20.

21. Iso H, Stampfer MJ, Manson JE, et al. Prospective study of calcium, potassium, and magnesium intake and risk of stroke in women. Stroke 1999;30:1772-9.

22. Hofman A, Grobbee DE, de Jong PT, et al. Determinants of disease and disability in the elderly: the Rotterdam Elderly Study. Eur J Epidemiol 1991;7:403-22.

23. Klipstein-Grobusch K, den Breeijen JH, Goldbohm RA, et al. Dietary assessment in the elderly: validation of a semiquantitative food frequency questionnaire. Eur J Clin Nutr 1998;52:588-96.

24. Dutch Food Composition Table (NEVO) 1993. Voorlichtingsbureau voor de Voeding. The Hague; 1993.

25. Van Gent CM, van der Voort HA, de Bruyn AM, et al. Cholesterol determinations. A comparative study of methods with special reference to enzymatic procedures. Clin Chim Acta 1977;75:243-51.

26. WHO. International statistical classification of diseases and related health problems, 10th revision, vol. 1. Geneva; 1992.

27. Barlow WE, Ichikawa L, Rosner D, Izumi S. Analysis of casecohort designs. J Clin Epidemiol 1999;52:1165-72.

28. Barlow WE. Robust variance estimation for the case-cohort design. Biometrics 1994;50:1064-72.

29. Liu K, Cooper R, McKeever J, et al. Assessment of the association of habitual salt intake and high blood pressure: methodological problems. Am J Epidemiol 1979;110: 219-26.

30. Bruce NG, Cook DG, Shaper AG, et al. Casual urine concentrations of sodium, potassium, and creatinine in population studies of blood pressure. J Hum Hypertens 1990;4:597-602.

31. Luft FC, Fineberg NS, Sloan RS. Estimating dietary sodium intake in individuals receiving a randomly functuating intake. Hypertension 1982;4:805-8.

32. MacMahon S, Peto R, Cutler J, et al. Blood pressure, stroke, and coronary heart disease, part 1: prolonged differences in blood pressure: prospective observational studies corrected for the regression dilution bias. Lancet 1990;335:765-74.

33. Geleijnse JM, Kok FJ, Grobbee DE. Blood pressure response to changes in sodium and potassium intake: a metaregression analysis of randomised trials. J Hum Hypertens 2003;17: 471-80.

34. Joint WHO/FAO Expert Consultation on Diet, Nutrition and the Prevention of Chronic Diseases (2002: Geneva, Switzerland). Diet, nutrition and the prevention of chronic diseases. WHO technical report series 916. Geneva: World Health Organization; 2003. 